\title{
On the evolution of neural decisions from uncertain visual input to uncertain actions
}

\author{
Alessandro Tomassini ${ }^{1 *}$, Darren Price ${ }^{1}$, Jiaxiang Zhang ${ }^{2}$, James B Rowe $e^{1,3}$ \\ 1. Medical Research Council Cognition and Brain Sciences Unit, University of Cambridge, CB27EF, \\ UK \\ 2. Cardiff University Brain Research Imaging Centre, School of Psychology, University of Cardiff, \\ CF2 2AT, UK
}

3. Department of Clinical Neurosciences, University of Cambridge, CB2 0SZ, UK

\footnotetext{
* Alessandro Tomassini Email: alessandro.tomassini@mrc-cbu.cam.ac.uk

Darren Price

Email: dprice80@gmail.com

Jiaxiang Zhang

Email: ZhangJ73@cardiff.ac.uk

James B. Rowe

Email: James.Rowe@mrc-cbu.cam.ac.uk
}

\section{Keywords}

Decision-making, evidence accumulation, Magnetoencephalography, effective connectivity.

\section{Author Contributions}

J.B.R and A.T. designed research; A.T. performed research; J.J. and D.P. contributed new reagents/analytic tools; A.T. analyzed data; A.T. and J.B.R. wrote the paper.

\section{Funding and disclosure}

This work was funded by the Medical Research Council and Wellcome Trust. The authors declare no competing financial interests and no conflict of interest.

Abstract (149 words) Main Text (4424 words) Methods (2909 words) Figures 1 to 7 References (70) Supplementary Appendix 


\section{Abstract}

1 Behavior can be conceived as the result of a sequence in which the outcomes of perceptual decisions

2 inform decisions on which action to take. However, the relationship between these processes, and

3 spatiotemporal dynamics of the visual-to-motor transformation remains unclear. Here, we

4 combined accumulation-to-threshold models and electro-magnetoencephalography, to trace neural

5 correlates of sensorimotor decisions in space, time and frequency. We challenge the assumption of

6 sequential decisions, with evidence that visuomotor processing unfolds through a continuous flow

7 of information from sensory to motor regions. Action selection is initiated before regional visual

8 decisions are completed. By linking behavior and physiology through theoretical decision models,

9 we identify simultaneous forward and backward flow of information for visuomotor decisions

10 between sensory and motor regions, in beta and gamma ranges. The model of integrated visuomotor

11 decisions provides a powerful approach to investigate behavioral disorders that impair the ability

12 to use sensory inputs to guide appropriate actions. 


\section{Introduction}

15 Human behaviors are the result of many decisions, from early or automatic perceptual inferences

16 about our environment to complex goal-directed choices between alternate courses of action. Three

17 broad lines of research have made separate contributions to understanding such decisions. First,

18 the psychophysical analysis of visuomotor task performance and reaction times, in health ${ }^{1}$ or in the

19 presence of focal ${ }^{2}$ and degenerative brain lesions ${ }^{3}$. Second, the functional anatomical analysis of

20 decision making using brain imaging and neurophysiology, including paradigms that manipulate

21 visual uncertainty ${ }^{4}$, action selection ${ }^{5}$, or outcome evaluation ${ }^{6}$. Third, the development of

22 computational models of how decisions can be reached, at the level of neuronal ensembles ${ }^{7}$ or

23 groups of individuals ${ }^{8}$.

It remains a challenge however, to bring these separate lines of enquiry together in a unified model of neurophysiologically informed decision process, embedded in a functional anatomical

27 framework, to explain the transformation of noisy visual inputs to alternative motor outputs. The

28 anatomical framework has an additional requirement, which is to accommodate the evidence for

29 functional segregation between sensory and motor areas at the same time as allowing the flow of

30 information through hierarchical and distributed brain networks. Here we propose an integrated

31 account of visuomotor decision-making, as summarised in Fig. 1, working from a novel visuomotor

32 task that adjusts sensory and action uncertainty during functional brain imaging by combined

33 electro-/magnetoencephalography (MEEG).

35 In mathematical psychology it has been argued that decisions and their latencies are controlled by when the cumulative evidence in favour of a choice reaches a criterion decision threshold ${ }^{9}$. We identify the accumulation-to-threshold of latent variables representing sensory evidences, based on the transformation of visual signals into evidence about the behaviorally relevant stimulus features (perceptual decisions ${ }^{10,11}$ ); and the analogous evidence for motor schema, which have been termed motor intentions (action decisions ${ }^{12,13}$ ).

42 Rather than arbitrarily divide visual from motor transformations, we investigated their associated 43 decision processes by separately manipulating uncertainty in the identity of visual features 44 (perceptual uncertainty ${ }^{11}$, by variable motion coherence) and range of possible actions (action 45 uncertainty $^{13,14}$, by variable number of response options). The task combined elements of the classic 
46 motion discrimination task ${ }^{11}$ with a response selection $\operatorname{task}^{13}$ : noisy visual stimuli indicated the one 47 or more response options, which were executed by pressing a corresponding button (Fig. 2). By 48 manipulating motion coherence in the random dots stimuli and the number of offered choices, we 49 sought to isolate the neural signatures of decision-evidence accumulation for perceptual and action 50 decisions, respectively.

51

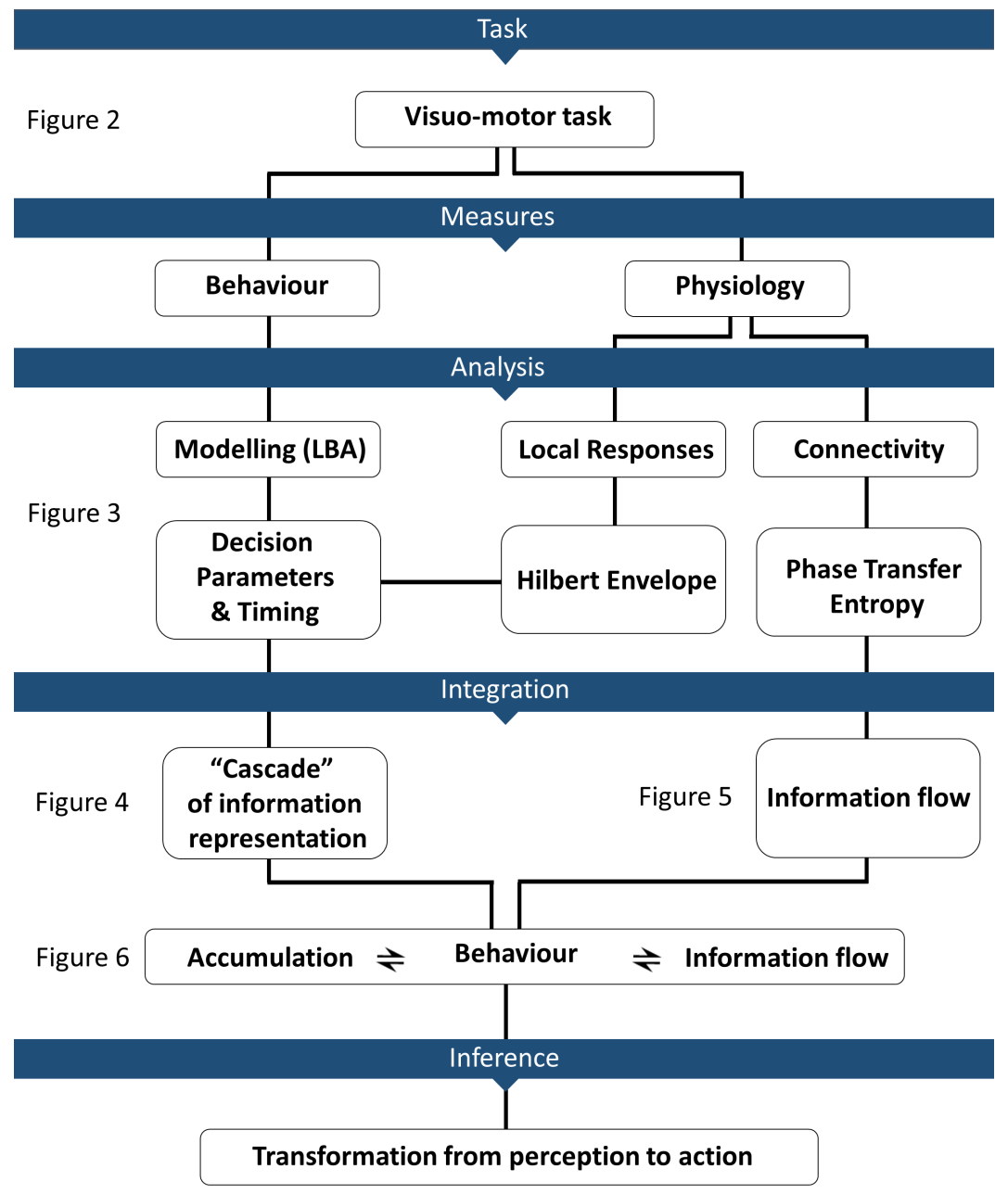

53 Figure 1. Overview of the study. We combined behavioural, computational, and neuroimaging approaches 54 to provide an integrated perspective of the decision processes linking perception to action. Each section is 55 expanded in a subsequent figure, as directed.

58 Several brain regions have been identified that accumulate perceptual evidence ${ }^{10,11}$ and motor 59 intentions ${ }^{12,13}$. However, it is necessary to understand how a network of accumulator regions 
60 orchestrates their activity for the critical transformation between perceptual and action decisions.

61 Specifically, we sought to distinguish (i) a serial process ${ }^{15}$ where perceptual decisions are complete

62 and their output passed to motor accumulators, from (ii) a continuous flow of information ${ }^{16}$, through

63 perceptual to associative and motor regions before completion of perceptual analysis. A serial

64 process would be robust to error, but continuous flow would enable faster action decisions. To

65 differentiate these alternatives, we mapped the modelled temporal profile of evidence accumulation

66 to MEEG induced power ${ }^{17}$, trial-by-trial. The temporal evolution of predicted evidence was based

67 on behaviorally optimized generative linear ballistic accumulator model of the decision.

68

69

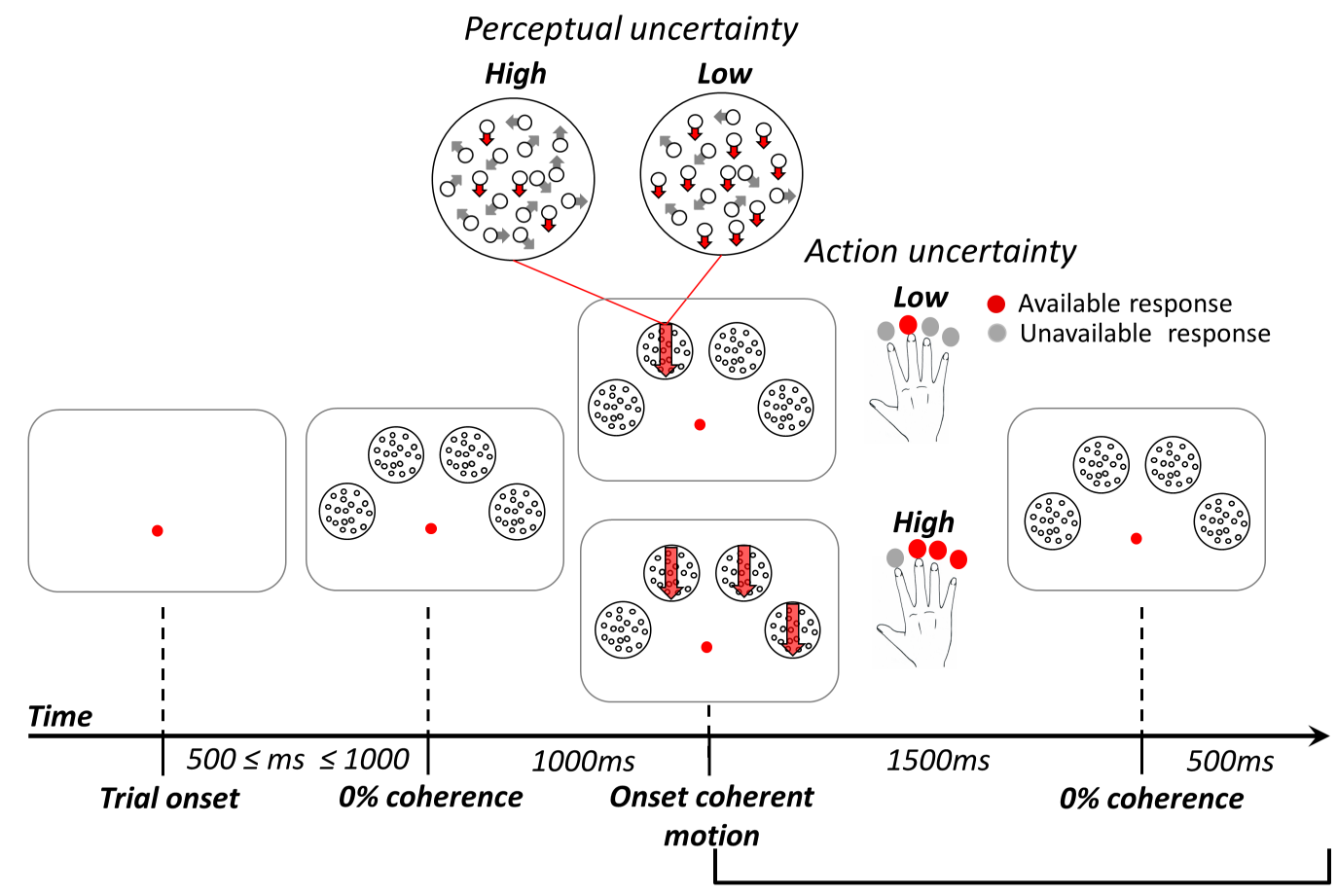

Button press

71 Figure 2. Experimental manipulation of perceptual and action uncertainty. Participants pressed the

72 button corresponding to the coherent stimulus (red downward arrow). When there were more than one

73 coherent stimulus, they selected one response and pressed the corresponding button. Perceptual uncertainty

74 was manipulated by changing the coherence of dot motion (i.e. by changing the motion strength), whereas

75 action uncertainty was manipulated by changing the number of available options (i.e. the number of coherent

76 stimuli to choose from). Perceptual and action uncertainty varied across trials in a 2 by 2 factorial design. 
78 We focused on the beta and gamma band power as the candidate correlates of the evidence for three

79 reasons. First, the growing evidence for separate functions of gamma and beta in the feedforward

80 and feedback of information respectively in hierarchical brain networks ${ }^{18}$. Second, that the

81 accumulation of evidence for perceptual choices correlates with gamma-frequency oscillations ${ }^{17}$.

82 Third, that the processes underlying the deliberation between alternate actions have been associated

83 with beta power modulation ${ }^{19,20}$.

84

85 The use of MEEG affords a source model of cortical generators ${ }^{21}$ and enables the functional 86 segregation of sensory and motor area, as well as areas where sensory-motor transformations occur.

87 Complementary connectivity measures (phase transfer entropy ${ }^{22}$ ) reveal the flow of information

88 between areas, orchestrating the emergence of decision-evidences across decision networks.

90 We show that evidence accumulation in motor and prefrontal cortex begins very soon after visual 91 cortex, and before perceptual decisions are concluded. We further demonstrate that the timing of 92 evidence accumulation and the direction of flow of information between widespread sensory, motor 93 and association cortices differ between Beta $(13-30 \mathrm{~Hz})$ and Gamma $(31-90 \mathrm{~Hz})$ frequency range. 94 An early sweep of Gamma activity across an occipito-parietal-frontal network precedes the gradual 95 arising of Beta mediated decision signals.

97 These signals emerge progressively in a lateralized caudo-rostral cascade unfolding along the 98 dorsal stream. The cascade is mainly driven by a continuous flow of information from posterior 99 visual areas to anterior action control regions. Crucially, the strength of the information flow (as 100 measured by phase-transfer entropy) determines the speed of progression throughout the stages of 101 information processing from perception through action as reflected by a positive relationship 102 between connectivity and both faster model accumulation-rates and shorter reaction-times. This 103 provides an important formal link between behaviour, established models of decision-making, and 104 connectivity measures. Taken together, the results reveal a continuous flow of information 
105

106

107

108

109

110

111

112

113

114

transmitted and integrated through a hierarchical network that transforms decision-making from perception to action.

\section{Results}

\section{Uncertainty modulates the speed of decisions}

Participants performed the task first in a training session where individual motion thresholds were estimated for both low and high action uncertainty levels (Fig. 3a). Subsequently, participants performed the task with the motion thresholds that standardized performance, while undergoing MEEG scan.

Behavioral performance scaled with levels of perceptual and action uncertainties, confirming the desired effect of task manipulations. During training, participants where slower and less accurate when motion coherence was lower (Fig. 3a). Similarly, during the scan session (Fig. 3b and SI Appendix, Fig. S1) responses were slower under high perceptual (low $=0.77 \mathrm{~s} \pm 0.1$; high $=$ $0.88 \mathrm{~s} \pm 0.1 ; \mathrm{F}(1,17)=158.17 \mathrm{p}<0.0001 ;$ post-hoc $\left.\mathrm{p}<0.0001 ; \eta \mathrm{p}^{2}=0.902\right)$ and action uncertainty $\left(\right.$ low $=0.80 \mathrm{~s} \pm 0.13 ;$ high $=0.85 \mathrm{~s} \pm 0.1 ; \mathrm{F}(1,17)=6.28 \mathrm{p}=0.022 ;$ post-hoc $\mathrm{p}=0.022 ; \eta \mathrm{p}^{2}=0.269 ;$ 2-by-2 repeated measures ANOVA; Tukey-Kramer correction).

Participants' choices were largely independent over trials as indicated by Shannon's equitability index (mean $0.77 \pm 0.016 \mathrm{SD}$, see SI Appendix and Fig. S2 for statistics) calculated for sequential choice pairs ${ }^{13}$.

\section{Uncertainty modulates the rate of evidence accumulation}

To decompose the behavioral performance into cognitively relevant latent variables, we fitted accumulation-to-threshold models (Linear Ballistic Accumulator ${ }^{23}$, LBA), to each participant's reaction time and accuracy data.

Uncertainty can slow responses by reducing the speed of information accumulation (accumulationrate), increasing response caution (decision boundary), stretching the time required by perceptual and motor processes not directly related to the decision process (non-decision time), or by a combination thereof. To differentiate these competing mechanisms, we fitted all possible 


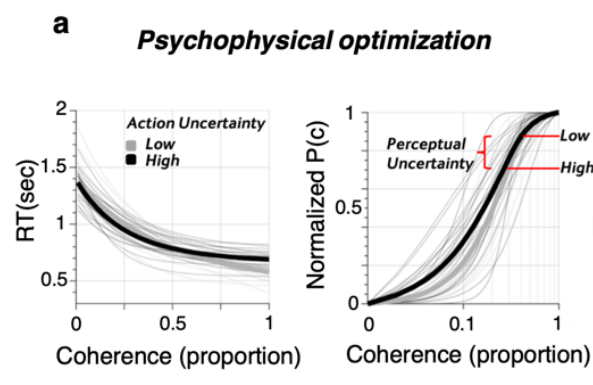

d
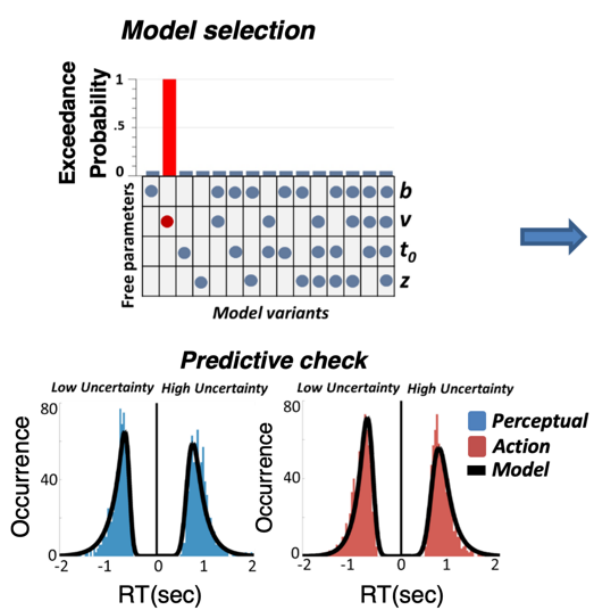

b RT experiment

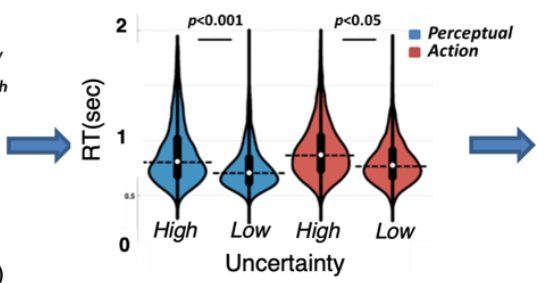

e Estimation model parameters

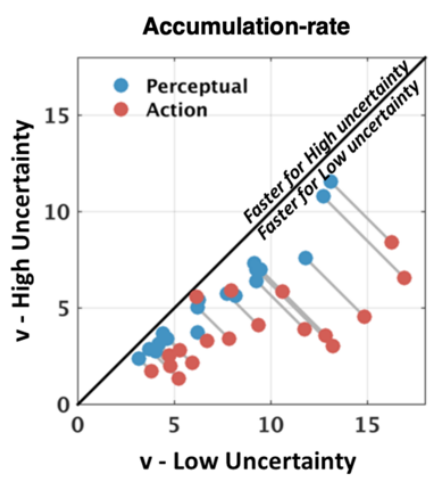

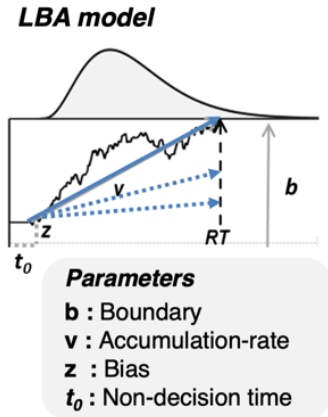

f

Trial-by-trial fitting to MEEG

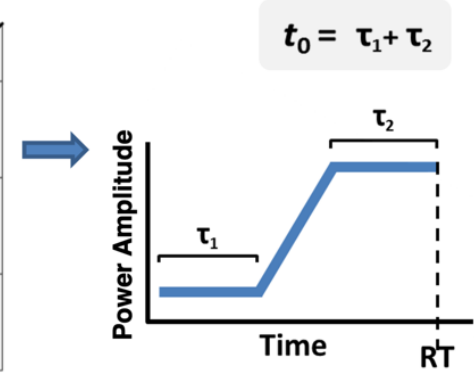

Figure 3. Uncertainty modulates reaction times and the speed of decision evidence accumulation. (a)

136

137

138

139

140

141

142

143

144

145

146

147

148

149

150

151

Decision evidence titrated to the participants' individual motion sensitivity. Reaction times (left panel) and accuracy (right panel) varied with motion strength (grey lines: individual data, black tick lines: mean data). Low and High perceptual uncertainty were estimated at the $75 \%$ and $90 \%$ accuracy levels, respectively. (b) During the experiment, reaction times were significantly modulated by both perceptual and action uncertainty (aggregated data for uncertainty type, rm-ANOVA on $\log (\mathrm{RT})$ ). (c) The task was modelled using a race accumulation model (Linear ballistic accumulator, LBA). Noisy evidence accumulates over time at a rate $v$ up to a decision bound $b$. The fastest accumulator (thick blue arrow) determines the choice. Non-decision time linked to sensorial and motor processes $\left(t_{0}\right)$ sums to the evidence accumulation time to produce reactiontimes. (d) Bayesian model comparison: changes in the sole drift-rate best accounted for the behavioral data. The quality of fit is also seen by the overlap of empirical reaction time distributions for each condition, with data simulated using the winning model. (e) The model predicted faster accumulation of decision evidence when uncertainty is low for both action and perceptual uncertainty (grey lines connects data points from each participant). (f) Model predicted activity was fitted to the power envelope of the MEEG signal in a trial-bytrial fashion to identify the latencies of accumulators of decision evidence. Non decision time $\left(t_{0}\right)$ was decomposed into pre-accumulation (taul) and post-accumulation (tau2) time reflecting perceptual and motor processes, respectively. 
152 combinations of free parameters in a set of 15 LBAs. We compared the goodness-of-fit of each 153 model using random-effects Bayesian model comparison ${ }^{24,25}$. The model comparison revealed that 154 changes in the accumulation-rate alone (model number 2; Fig. 3d top panel) accounted 155 parsimoniously for the effects of uncertainty on behavior. The goodness-of-fit of the winning model 156 was further confirmed by posterior predictive checks (Fig. 3d bottom panel), performed by 157 simulating data under the winning model and then comparing these to the observed data.

158 In the winning model (henceforth, the LBA model), high uncertainty is associated with 159 comparatively slow accumulation rates. This relationship between uncertainty and accumulation 160 rate held for both perception $(\mathrm{z}=3.723, \mathrm{p}=0.00019$; Wilkoxon sign rank test $)$ and action $(\mathrm{z}=$ $1613.723, \mathrm{p}=0.00019$; Wilkoxon sign rank test) uncertainty, as well as for each subject (Fig. 3e), in 162 accord with previous studies ${ }^{11,14}$.

163 Non-decision time ( $\left.t_{0}\right)$, encompassing sensory delays and motor execution, was estimated to be $164370 \mathrm{~ms}$ on average (see SI Appendix, table S1), which is within the plausible range of non-decision 165 times for humans ${ }^{26}$.

\section{Localization of decision-evidence accumulation}

168 The temporal evolution of the combined MEG and EEG spectral power (power envelope) from each region of interest (ROI) of the parcellated cortical surface served as the signal for our analysis in beta $(13-30 \mathrm{~Hz})$ and gamma $(31-90 \mathrm{~Hz})$ bands. The time onset of evidence accumulation across

171 ROIs was identified by optimizing the split of the non-decision time before and after the 172 accumulation period using Spearman correlation to the single-trial MEEG power envelope (Fig. 173 3f, see Methods and Materials for details, supplemental Fig. S3 for the statistical map). This 174 allows one to depict in space and time the emergence of decision-evidence accumulation.

175 In support of our hypothesis that both increasing and non-increasing activity can mediate evidence 176 accumulation $^{27,28}$, we found significant (negative) correlations between the LBA model predictions 177 and the MEEG oscillations in beta and gamma bands ${ }^{17}$ (Fig. 4). Specifically, for both beta and 178 gamma, neural activity after coherence onset desynchronized in a graded fashion and peaked 179 approximately before response suggesting a form of threshold mechanism (Fig. 4a) ${ }^{29,30}$. our predictions. As the decision unfolds, the accumulated decision-evidence will ramp quickly with 
182 low perceptual uncertainty, and slowly with high perceptual uncertainty. Accordingly,

183 desynchronization of beta power-envelopes averaged across trials and ROIs was larger $(\mathrm{p}<0.0001$, cluster corrected random permutations) for low than high perceptual uncertainty ${ }^{18,29}$.

185 When a response is chosen between multiple options, the race underlying the selection of each

186 alternative is characterized by an overall larger amount of decision-evidence summed across all he 187 racing accumulators by the time of response ${ }^{13,14}$. Accordingly, desynchronization of beta powerenvelopes averaged across trials and ROIs was larger for high than low action uncertainty $(\mathrm{p}<$ 0.0001, cluster corrected random permutations). Gamma power-envelopes, showed a similar trend,

190 but the effects were statistically insignificant.

191 Decision-related dynamics expressed by beta desynchronization were distributed across a wide 192 network (Fig3b, mean across significant ROIs: sign-test $z=-3.15 \pm 0.48, p=0.00065 \pm 0.0016$, 193 FDR corrected) similar to previous EEG reports ${ }^{31}$.

194 In the gamma band we observed a more localized mosaic of ROIs including contralateral motion 195 sensitive areas (inferior lateral occipital region), bilateral extrastriate areas and bilateral frontal 196 motor regions (comprising premotor areas and supplementary motor area; mean across significant 197 ROIs: sign-test $\mathrm{z}=-2.27 \pm 0.27, \mathrm{p}=0.0058 \pm 0.003$, FDR corrected). manipulations in isolation confirmed that the quality of fit and results did not vary across trials types ( $>0.05$, FDR corrected).

202 We traced the spectrally resolved temporal evolution of decisions through the visuo-motor 203 hierarchy, finding that decision-evidence accumulation emerges with distinct spatio-temporal 204 profiles between beta and gamma (Fig. 4b). An early wave of accumulation begins at $\sim 120 \mathrm{~ms}$ from 205 coherence onset within the sparse network oscillating at gamma frequency. It is followed by a 206 second wave mediated by Beta at $\sim 160 \mathrm{~ms}$ from coherence onset (Fig. 4c; Conjunction of significant 207 ROIs in beta and gamma, median latency across participants, $\mathrm{z}=5.53, \mathrm{p}<0.0001$, Wilkoxon rank 208 test). No difference in latencies was found between hemispheres across frequency bands. 


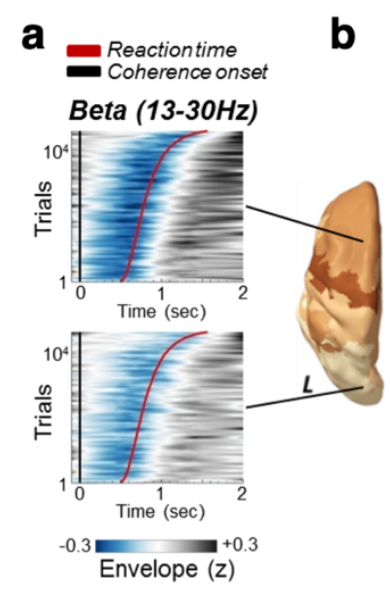

Gamma (31-90Hz)
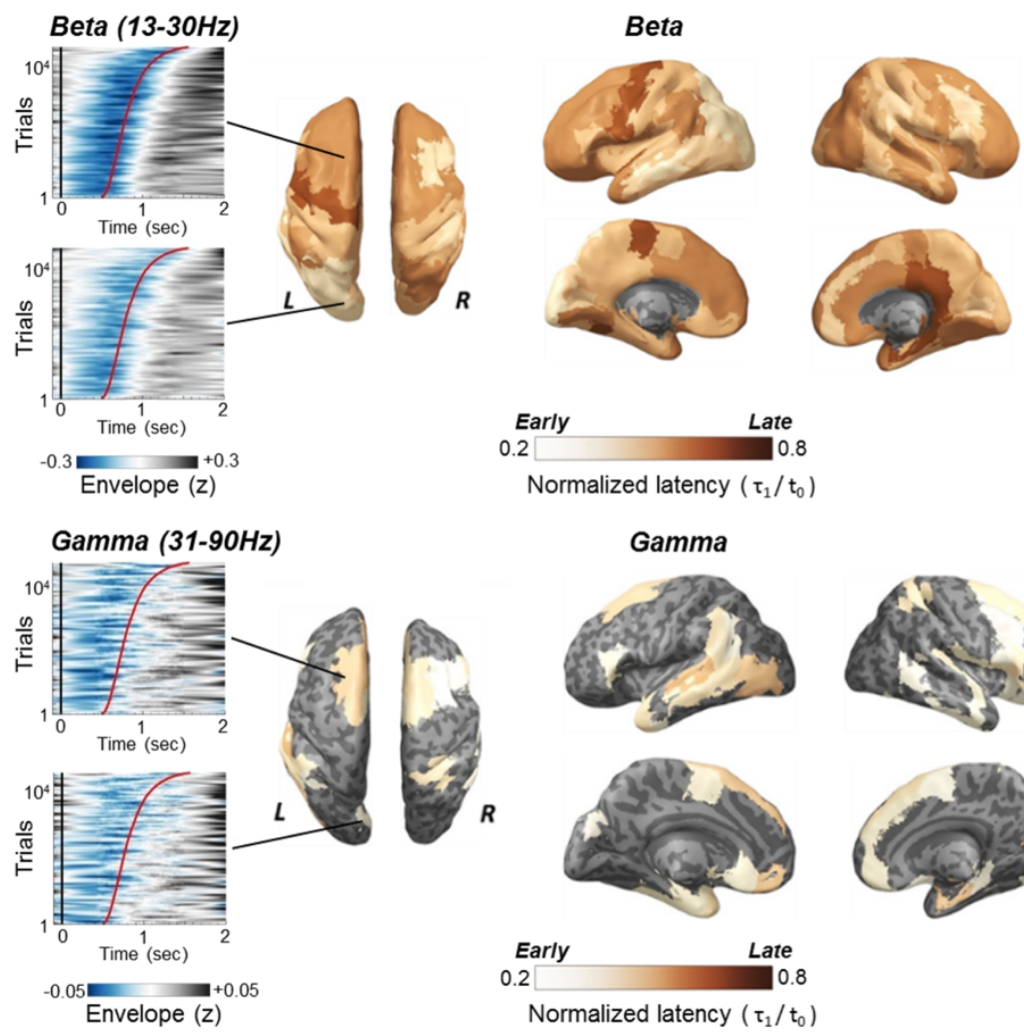

Normalized latency $\left(\tau_{1} / t_{0}\right)$
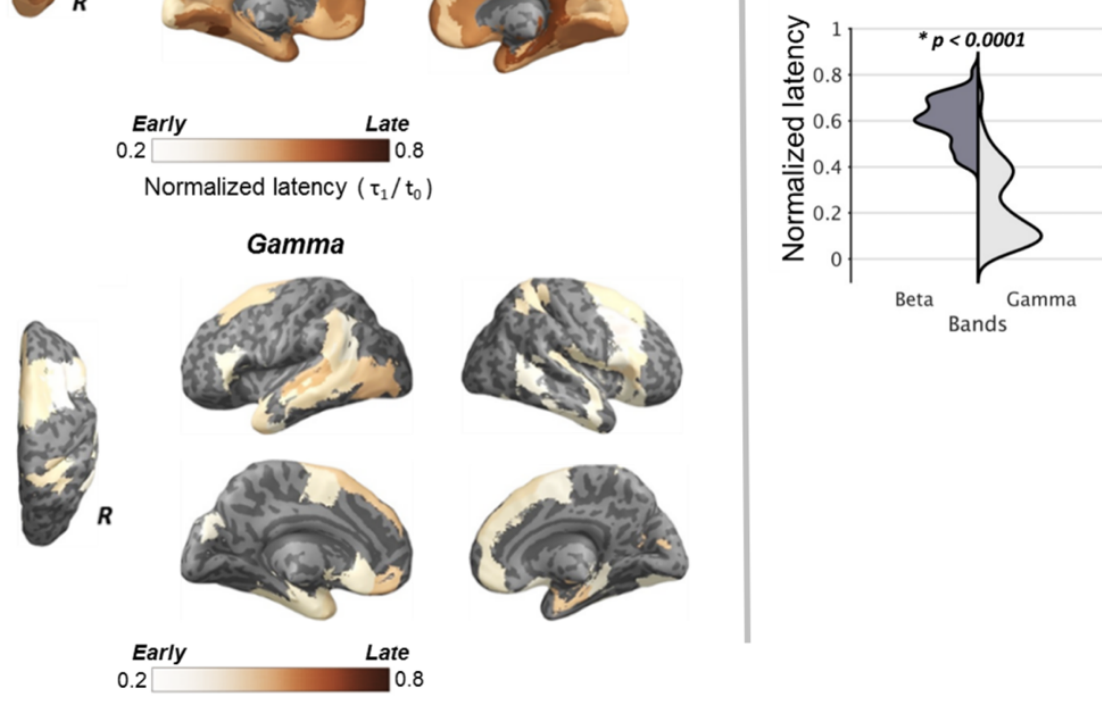

Figure 4. Temporal cascade of decision-evidence accumulation revealed by comparing trial-by-trial

MEEG power envelopes to model's predictions. (a) Power plots ranked by reaction-times showing the temporal relationship between signal power envelope (z-scored with regard to each individual's baseline average) and reaction times (red curve) at representative ROIs showed separately for Beta band (top row) and gamma band (bottom row). The ordinate of each plot represents individual trials pooled across participants and sorted according to reaction times. The black line indicates motion coherence onset. (b) Latency maps showing the normalized latencies (each accumulation onset time divided by individual nondecision time) of decision-evidence accumulation across anatomical regions where correlations between power-envelopes and model's predictions survived random permutation testing (grey areas denote not significant correlation). (c) Decision-evidence accumulation in the gamma band precedes beta (top panel).

222 The latency maps (Fig. 4b) show an accumulation gradient towards the precentral gyrus. We fitted

223 a piecewise regression model with a free internal knot to the mean latencies of ROIs located along 224 the dorsal path (Fig. 5a), a critical system for visuomotor decisions ${ }^{32,33}$. In keeping with our observations the model (Fig. 5b left top-bottom panels) identified the precentral gyrus (comprising 
226 primary motor cortex and part of the premotor cortex) as the point of convergence of two linear 227 functions $\left(\mathrm{R}^{2}=0.734, \mathrm{p}<0.0001\right)$ and outperformed a single regression model (piecewise $\mathrm{R}^{2}$ adj $=$ 2280.681 ; linear $\mathrm{R}_{\text {adj }}^{2}=0.649$; adjusted $\mathrm{R}^{2}$ penalizes extra free parameters in favor of simple models).
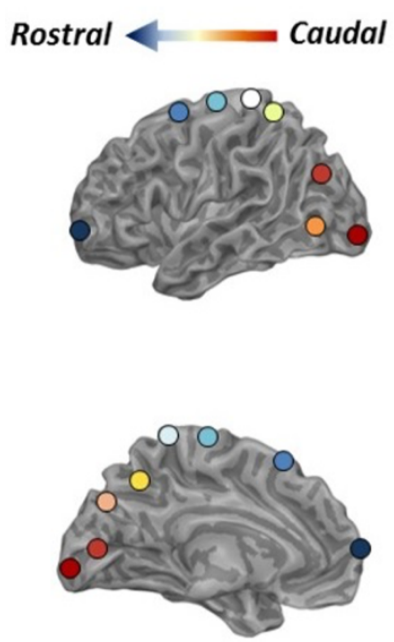

Caudal
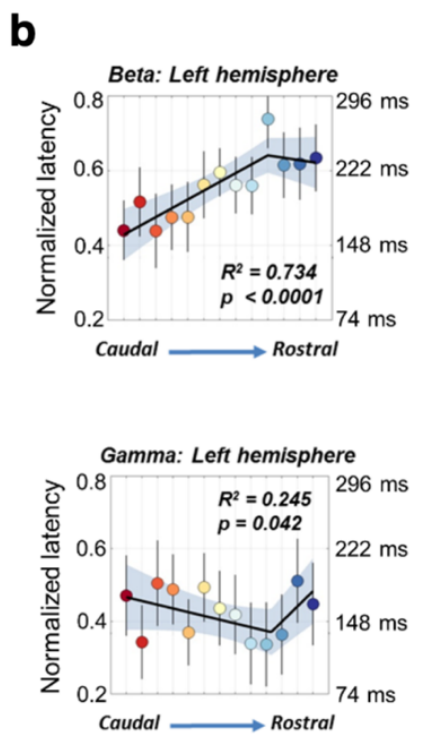
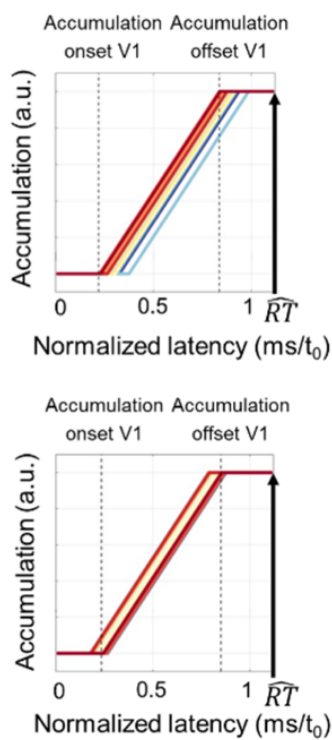

Figure 5. A quasi-parallel cascade of decision-evidence accumulation unfolds along the caudo-rostral axis of the dorsal path (a) Regions of interest (ROIs) along the dorsal path color-coded with respect to their position along the caudo-rostral axis (b) Decision-evidence accumulation mediated by beta follows a caudorostral gradient along the dorsal path of the contralateral hemisphere. A piecewise regression (top left panel) best describes the gradient showing that latencies increase from visual areas up to M1 in the precentral gyrus and decrease afterwards suggesting two separate converging flows (Error bars indicate SEM, shaded area covers bootstrapped $95 \%$ regression CI). The pattern is inverted for gamma where latencies increase while proceeding from M1 to posterior and anterior ROIs. Despite the differences in latencies along the gradient, the cascade of decision-evidence accumulation is quasi-parallel. The right panels show that the latest ROI in the gradient starts accumulating decision-evidence before the earliest ROI (e.g. V1 for beta) has reached the decision boundary. RT hat is the mean reaction time (across trials and participants) normalized by the mean non-decision time $\left(t_{0}\right)$.

Interestingly, in the gamma band (Fig. 5b bottom left panel) we found a mirror-symmetric trend with increasing accumulation latencies while proceeding from the precentral gyrus to more

244 posterior and anterior regions $\left(\mathrm{R}^{2}=0.245, \mathrm{p}=0.042\right)$. Thus, accumulation starts with gamma at

$245 \sim 120 \mathrm{~ms}$ from coherence onset in the precentral gyrus and at $\sim 160 \mathrm{~ms}$ in the occipital and frontal poles. 
247 The onset of the accumulation in beta overlaps with gamma in the occipital pole at $\sim 160 \mathrm{~ms}$ from

248 coherence onset ${ }^{34}$. The interval from earliest onset of accumulation to last onset, is only $\sim 100 \mathrm{~ms}$

249 and the onset in precentral gyrus is on average $\sim 570 \mathrm{~ms}$ before a motor respons $\mathrm{e}^{35}$. The delay from

250 motion onset to the beginning of the accumulation on the occipital pole $(\sim 160 \mathrm{~ms})$, and the delay

251 from action decision to movement initiation in precentral gyrus $(\sim 100 \mathrm{~ms})$ are close to the sensory

$252(\sim 200 \mathrm{~ms})$ and motor $(\sim 80 \mathrm{~ms})$ delays measured from neural recordings on macaque ${ }^{11,36}$.

253 These patterns, albeit with lower spatial resolution, were also found at the sensor level (SI

254 Appendix, Fig. S4). As a note of caution for the piecewise regression, the fit of the LBA model

255 for some of the ROIs within the dorsal path was not significant in the gamma band, reducing the

256 accuracy of their latency estimates.

257 An important observation is that the latest ROIs in the gradient for both beta and gamma starts

258 accumulating decision-evidence before the earliest ROI (e.g. the occipital lobe for beta) has reached

259 its decision boundary (Fig. 5b right top-bottom panels). This suggests that decisions are made on

260 the basis of a continuous flow of information, rather than a serial sequence of discrete decisions. 
262 The above analyses identified a flow of information across a widespread visuomotor network. To

263 functionally segregate accumulators sub-serving perceptual and action decisions, and to reveal the

264 influx and efflux of information across them we measured the phase-transfer entropy, a data-driven measure of information flow that is robust to signal leakage ${ }^{22}$.

266 Fig. 6a shows, for the beta band, the regions modulated by action uncertainty (Action decision regions, $\mathrm{p}_{\text {corrected }}<0.0005$ in all ROIs) and perceptual uncertainty (Perceptual decision regions, $268 \mathrm{p}_{\text {corrected }}<0.0005$ in all ROIs). Action decision regions include ipsilateral cingulate and 269 paracingulate cortex ${ }^{37}$, contralateral frontopolar cortex, ventromedial cortex, insula, supplementary 270 motor cortex, inferior parietal lobule and medial parietal cortex ${ }^{13,38}$. Of notice, bilateral precentral 271 gyri were identified as action decision regions which replicates previous findings ${ }^{13,30}$.

272 Perceptual decision regions in the contralateral hemisphere include posterior areas typically 273 associated with decisions about motion direction. These include lateral occipital cortex (including 274 motion area MT-complex), superior temporal cortex (comprising the superior temporal sulcus) and 275 the superior parietal lobule comprising the superior intraparietal sulcus ${ }^{39}$ along with the 276 dorsomedial frontal cortex ${ }^{4,40}$. Interestingly, two areas along the dorsal path on the left hemisphere 277 were sensitive to both perceptual and action uncertainty manipulations (superior frontal gyrus, 278 middle frontal gyrus, lateral occipital cortex superior division (comprising V2 and V3; $\mathrm{p}_{\text {corrected }}<$ 2790.0005 in all ROIs).

280 In the gamma band, we observed bilateral involvement of the superior frontal gyrus ${ }^{41}$ and inferior 281 frontal gyrus pars triangularis ${ }^{5}$, along with contralateral frontal medial cortex ${ }^{13}$ and ipsilateral 282 paracingulate gyrus in action decisions ( $\mathrm{p}_{\text {corrected }}<0.005$ in all ROIs). Perceptual decision areas 283 ( $\mathrm{p}_{\text {corrected }}<0.0005$ in all ROIs) included bilateral superior temporal areas (comprising the superior 284 temporal sulcus $^{39}$ ), cuneal cortex, and subcallosal cortex which has been linked to early encoding 285 of confidence for perceptual decisions ${ }^{42}$.

286 The dominant direction of information transfer between ROIs was estimated using the directed 287 phase-transfer entropy ${ }^{22}$. The average direction of information flow for each ROI was computed 288 resulting in a single estimate of preferred direction of information flow (either inflow or outflow).

289 Based on these estimates, we calculated a posterior-anterior index ${ }^{22}$ (PAx) to quantify the direction 290 of flow between caudal and rostral ROIs. 
291 Fig. 6b show the smooth global pattern of preferential information flow in the beta range with caudal ROIs preferentially sending information to anterior regions. This pattern is similar to that reported previous work in human resting $\operatorname{state}^{22}$, except that our results show a task-related lateralization, with the contralateral PAx almost twice the size of the ipsilateral one (left: $\mathrm{p}=$ 0.0001, PAx $=0.357$; right: $\mathrm{p}=0.0083 ; \mathrm{PAx}=0.184$; left vs right $\mathrm{PAx}, \mathrm{p}<0.0001$ ).

Beta $(13-30 H z)$

a

$L$

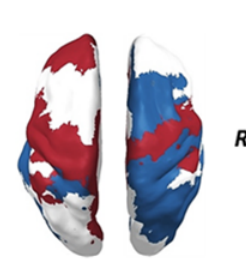

b

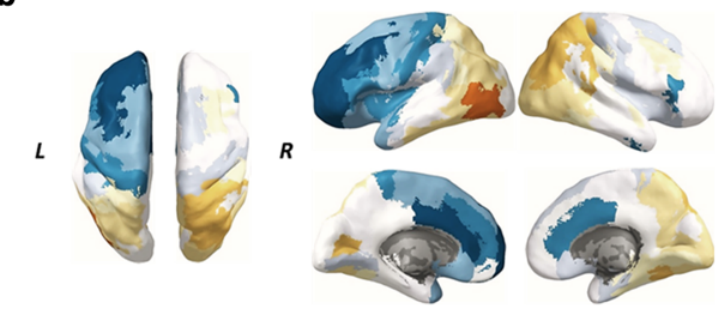

C

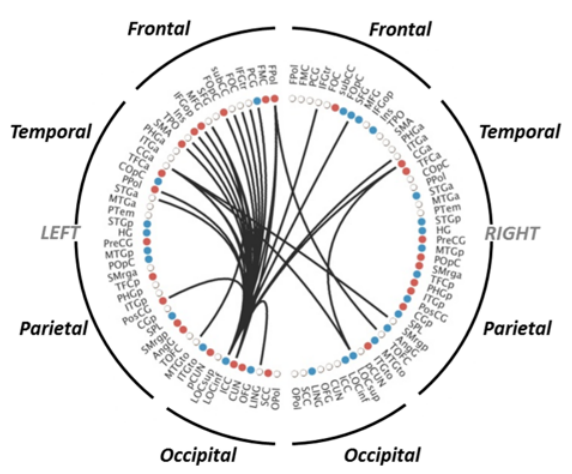

Gamma (31-90Hz)
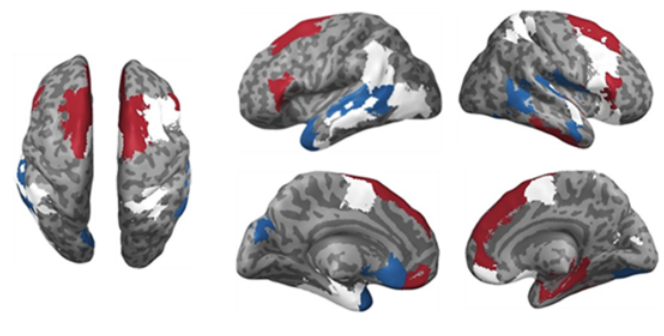

Perceptua

Action

Both (N)

Neither
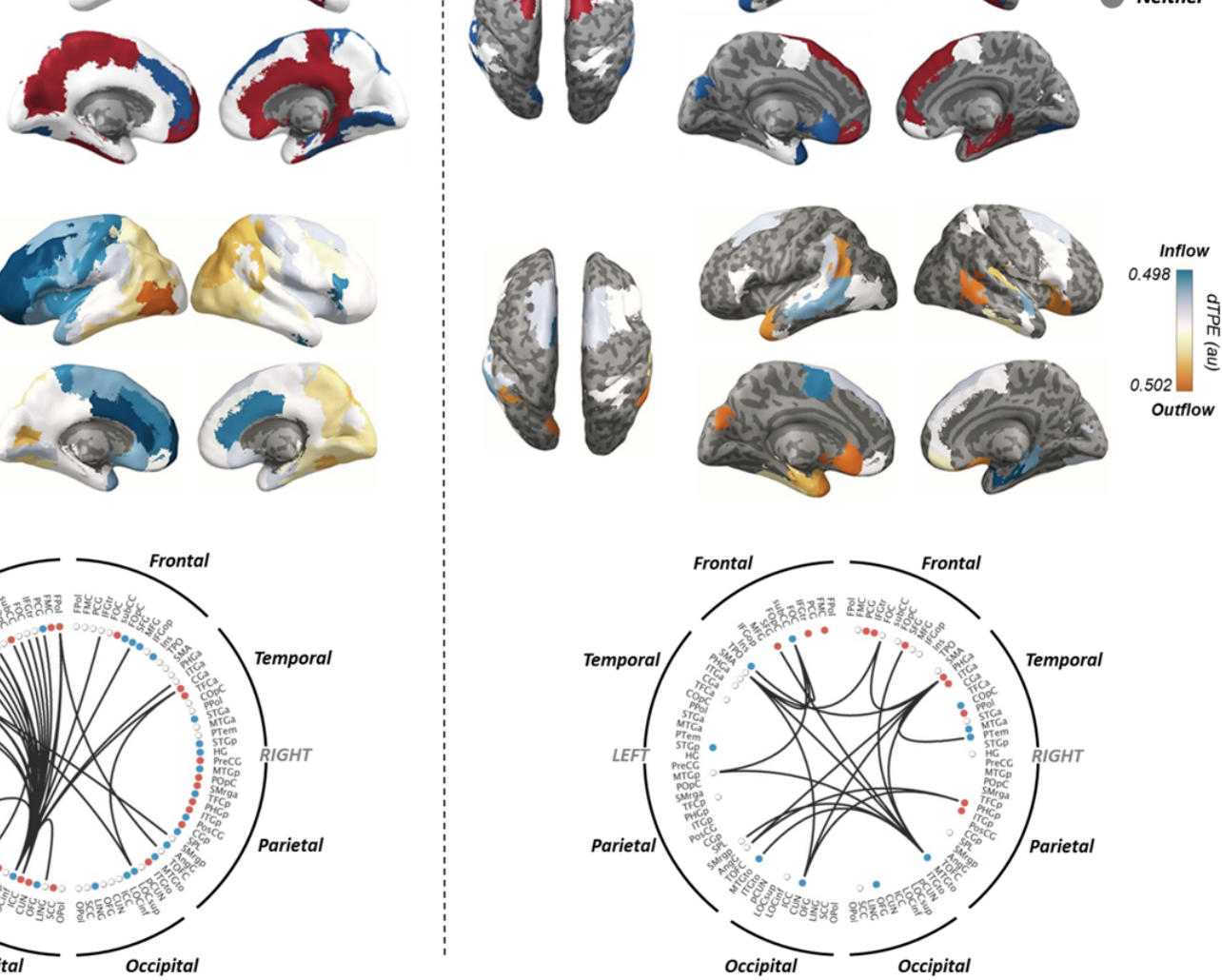

Figure 6. Sensitivity to uncertainty and information flow, show distinct spatiotemporal gradients. (a)

Differences in phase transfer entropy between manipulations of perceptual and action uncertainty for beta (left column) and gamma (right column) allows to define regions accumulating information specific to perception and action decision. (b) Information flow (directional phase transfer entropy) shows a clear rostrocaudal gradient in beta with MT-complex and the frontal regions being the strongest sender and receiver of information across distant cortical regions mostly within the left hemisphere. Gamma shows a more local activity with no clear lateralization. The full names of the ROIs are given in Table S2. 
306 It can be seen from Fig. 6b-c that, for beta, the strongest information flow was from the left lateral 307 occipital cortex to the left middle frontal gyrus and the frontopolar cortex. This accords with 308 previous reports of beta-synchronization between primate MT and frontal regions during motion 309 discrimination ${ }^{43}$. No significant preferential flow from posterior to anterior regions was seen for 310 the gamma range in either hemisphere (left $\mathrm{p}=0.79 ; \mathrm{PAx}=0.05$; right $\mathrm{p}=0.37$; $\mathrm{PAx}=0.3$ ) which

311 is expected given the pattern of accumulation latencies observed in gamma but might also reflect 312 the shorter range of gamma interactions ${ }^{44}$.

\section{Integration of behavioral, computational and physiological evidence}

314 To highlight the behavioral relevance of the integrated account of visuomotor decision-making, we 315 explored the relationships between connectivity, accumulator model parameters and behavior. To 316 account for multiple-comparisons, we used Holm-Bonferroni correction over eight tests.

317 In the beta range, the caudo-rostral gradient of evidence-accumulation is matched by a gradual 318 transition from perception to action decisions, as shown by a positive correlation between regional 319 specificity to the type of uncertainty and the estimated accumulation latencies (Fig. 7a top left 320 panel, $\mathrm{r}=0.27, \mathrm{p}_{\text {corrected }}=0.044,95 \%$ CI $\left.[0.0790 .420]\right)$. Moreover, the information flow is aligned 321 with the caudo-rostral gradient of accumulation since the flow proceeds from perceptual-decision 322 regions to action-decision regions (Fig. 7a bottom left panel, correlation between regional 323 specificity and direction of information flow: $\mathrm{r}=-0.37, \mathrm{p}_{\text {corrected }}=0.0016,95 \% \mathrm{CI}[-0.555-0.203]$ ).

324 In contrast, for the gamma band we found there was neither significant relationship between region 325 specificity and accumulation latency (Fig. 7a top right panel, $r=0.19, p_{\text {corrected }}=0.759$ ) nor 326 significant evidence of flow of information from perception to action decision regions (Fig. 7a 327 bottom right panel, $\mathrm{r}=-0.37, \mathrm{p}_{\text {corrected }}=0.068$ ).

328 Finally, we hypothesized that in a continuous flow of information the amount of information 329 transferred between perceptual and action accumulators co-varies with the rate of accumulation. 330 Faster progression from perception through action should be correlated with phase transfer entropy and model accumulation rate, but negatively with reaction-times. 
332 This was the case in the beta range where strong flow was associated with short reaction times

333 (Fig. 7b top left panel, repeated-measures correlation: $\mathrm{r}_{\mathrm{rm}}=-0.378, \mathrm{p}_{\text {corrected }}=0.03,95 \% \mathrm{CI}[-0.588$,

$334-0.12]$ ) and accumulation rates (Fig. 7b bottom left panel, $\mathrm{r}_{\mathrm{rm}}=0.356, \mathrm{p}_{\text {corrected }}=0.045,95 \% \mathrm{CI}$

$335[0.096,0.572])$. No significant correlation was observed in gamma (Fig. 7b top-bottom right,

336 reaction times vs information flow: $r_{\mathrm{rm}}=-0.079 \mathrm{p}_{\text {corrected }}=0.564$; accumulation-rate vs information

337 flow: $\mathrm{r}_{\mathrm{rm}}=0.113$, $\left.\mathrm{p}_{\text {corrected }}=0.816\right)$.

a

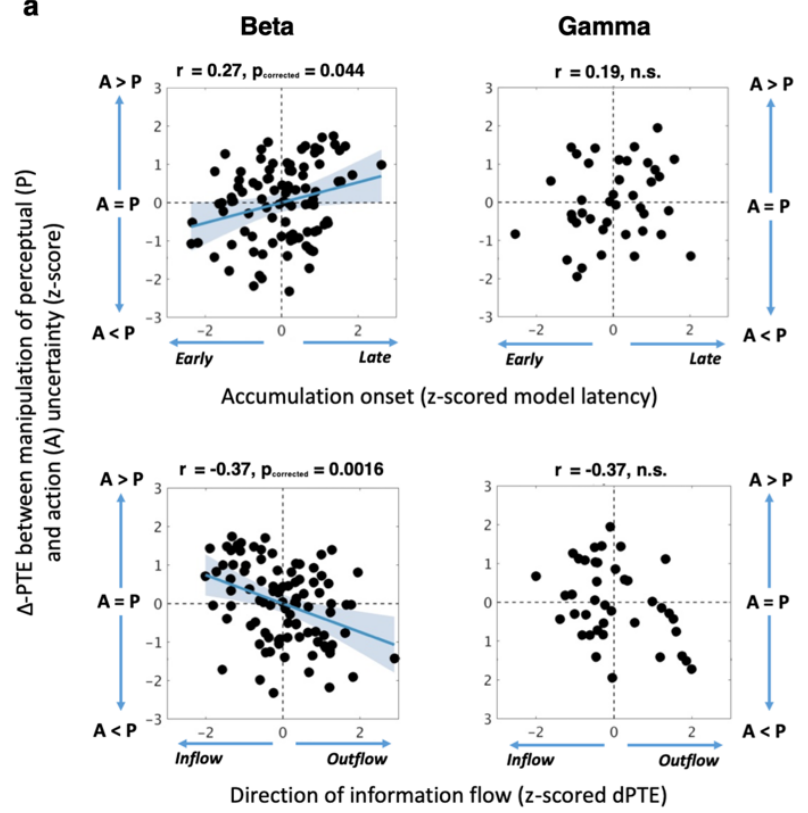

b
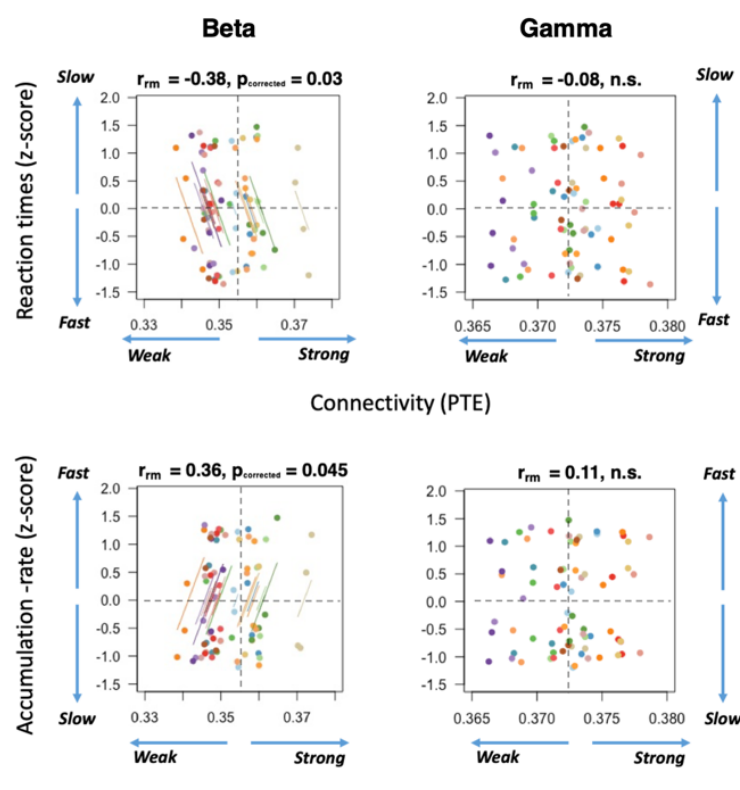

Connectivity (PTE)

Figure 7. Information flow is stronger under low uncertainty and associated with faster reaction times latency (top row) and direction of information flow (bottom row). Perceptual regions tend to show shorter latencies and to drive information transmission more than action regions. Points show z-scores of median values across subjects for each ROI (96 beta ROIs, 40 gamma ROIs). Shaded area covers bootstrapped 95\% CI. (b) Repeated-measures correlations. Reaction times (top-row) and accumulation-rates (bottom row) correlated with phase transfer entropy in the beta (left column) and gamma (right column) band. Points show median values for the four tested conditions. Data from the same participant are displayed in the same color, with the corresponding lines showing the individual fit of the repeated-measures correlation 
350

351

352

353

354

355

356

357

358

359

360

361

362

363

364

365

366

367

368

369

370

371

372

373

374

375

376

377

378

379

380

381

382

383

384

385

\section{Discussion}

There are two principal results from this study that illuminate the interaction between neural systems for perception and action. First, decisions in regions sensitive to motor precision do not wait until regional sensory decisions are completed. Instead, the accumulation of evidence in motor decisions begins within $100 \mathrm{~ms}$ after the initiation of evidence accumulation in the early sensory regions. This indicates a continuous flow or cascade of information and its gradual transformation from sensory evidence to motor intention ${ }^{45}$. Second, the correlates of evidence-accumulation in the beta and gamma frequency ranges have distinct spatiotemporal profiles, and opposite dominant directions of flow. This spectral directionality is predicted by hierarchical cortical networks for prediction and inference in visuomotor control ${ }^{18,46-48}$. In the beta band, there is not only a spatial gradient in the timing of accumulation-to-threshold between occipital and pre-central cortex, but also a qualitative change in the accumulated signals: from sensitivity to visual uncertainty to sensitivity to response uncertainty. Variability in the strength of the beta-mediated flow of information connecting visual to motor areas, accounts for participants' decision speed and corresponds to variability in the evidence accumulation rate (Fig. 7b). This relationship points to the strength of information flow as a neurophysiological correlate of the speed of evidence accumulation towards a decision.

We set out to integrate the analysis of information flow with decision-making implemented by the accumulation of evidence, and assess their joint influence on trial-to-trial variation in behavior (see Fig. 1). Independent manipulation of perceptual and action uncertainty was coupled with the decomposition of performance into latent variables in a parsimonious linear ballistic accumulator model ${ }^{23}$, which accurately generated the response distributions in each task condition including the expected effects of task variance on response latencies ${ }^{11,14}$. The model predictions of within-trial accumulation were correlated with change in beta and gamma power after the onset of stimulus coherence. Beta desynchronization has been shown to scale with uncertainty ${ }^{30}$, but here we show its interaction with the temporal evolution of decision making over sub-second intervals. The observed desynchronization displays two signatures of the accumulation-to-threshold class of models: accumulation of decisionevidence over time and the consistent bound reached shortly before each movement ${ }^{10,29,35}$.

Beta and gamma desynchronization are correlated with behavioural performance. For example, in direct recording from non-human primates during working memory ${ }^{49}$ and sensory discrimination ${ }^{50}$, the beta band desynchronization was greater for accurate trials compared with inaccurate trials. Such beta power encoding of decision outcomes is supramodal in many cortical areas ${ }^{20}$. The change in beta power followed the change in gamma power as in the current study: we found an early wave of gamma followed by a second wave of beta. 
Although gamma and beta rhythms have been observed to occur together or in close succession ${ }^{51,52}$, the temporal relationship is functionally relevant. For hierarchical cortical networks, message passing between regions is a function of the laminar asymmetry of afferent vs. efferent connections ${ }^{46}$, and the properties of columnar circuitry which preferentially generates gamma rhythms superficially, and lower control $^{18,48}$.

400

The spatial gradient of gamma latencies is reversed, with earliest changes observed in precentral cortex, before occipital cortex, and later gamma latencies in time with beta responses in occipital cortex. This may be because of the difference between predicting when a response may be required and what that response should be $\mathrm{e}^{55}$. The sensory stimulus change (visual coherence) in our task is not the result of the participant's own response, but is predictable a second after the onset of the non-coherent display. The participant can predict when an action is required, but not which actions are permitted or specified. An increase in localized and predominantly short-range interactions in gamma range may therefore be a permissive of information required for the beta-mediated decision between action alternatives ${ }^{56}$.

Despite the similar latency of beta and gamma accumulators in occipital cortex, the connectivity analyses indicate distinct routing information at longer and shorter spatial scales, respectively. The pattern of net efflux $v s$. influx of beta (Fig. 6b) shows a clear division between frontal cortex and posterior lobes. Moreover, the more sensitive a region is to action uncertainty ( $v s$. perceptual uncertainty), the later its onset of beta accumulation, and the greater its bias to inflow (vs. outflow, Fig. 7a). In other words, there was a cascade of overlapping accumulators and information flow along a the response hand. also been related to a dynamic decision process with updating of a motor plan as a decision evolves $^{29,30,35}$. The beta power lateralization in motor areas was correlated with the state of decision- 
422

423

424

425

426

427

428

429

430

431

432

433

434

435

436

437

438

439

440

441

442

443

444

445

446

447

448

449

450

451

452

453

454

455

456

457

evidence. Crucially, these earlier MEG and EEG studies used a fixed-mapping between decisions outcomes and categorical behavioural responses, without choice or independence of perception and action decisions. When this fixed mapping between perceptual decisions outcome and motor responses is removed, sensorimotor beta lateralization disappears ${ }^{57}$. Our findings complement this work by directly revealing a lateralized progression of evidence accumulation from posterior perceptual regions to anterior motor areas.

Previous work on visuomotor decisions has often focused on processes occurring at the final choice stage, leaving unresolved the question of whether evidence accumulation is coordinated throughout the cortex or in specific regions. Our findings support a generalized model in which accumulation-tothreshold provides a canonical mechanism evolving throughout all layers of a visuomotor transformation (Fig. 3a) and suggest that evidence accumulation is not a limited (perceptual) process with a single cortical focus, but is distributed ${ }^{58,59}$ and applicable to non-sensory evidence or intentions. This multi-focal property of evidence accumulation resonates with results from animal optogenetic ${ }^{58}$ and pharmacological ${ }^{59}$ studies showing that inactivation of local cortical areas carrying decision-related activity did not affect decision-making performance.

Taken together, our observations suggest that the beta band response links sensory evidence to motor plans, throughout a widespread network ${ }^{60}$. We propose that an early neural signalling regarding the need for a response is followed by a second phase that integrates a continuous flow of information to make a decision between them ${ }^{61}$. In this second phase, decisions unfold on the basis of a continuous flow of information (Fig. 5b), rather than sequential completion of intermediate decisions at the population level. However, this hypothesis refers to the population level, and we cannot exclude the possibility that within each region, a subsection of neurons completes the relevant decision and forwards this outcome to the next level in the hierarchy, while others in that region continue to accumulate.

The fluctuations in the strength of information flow caused by changes in uncertainty are behaviourally relevant, in their positive correlation with accumulation-rate and negative correlation with reaction times. This establishes an important formal link between behaviour, models of decision-making, and physiological connectivity: Fast accumulating-rates of the linear ballistic accumulator model are associated with a more effective beta-mediated information flow throughout the visuo-motor processing hierarchy, resulting in faster decisions and responses (Fig.7). This link provides a powerful tool to investigate clinical conditions in which the ability to use sensory inputs to guide actions is impaired. Examples include Progressive Supranuclear Palsy, Parkinson's disease and functional movement disorders, which are associated with abnormal evidence accumulation rates ${ }^{62,63}$, and abnormal beta power ${ }^{64,65}$. 
459 In summary, our analytical approach set out in Fig 1 elucidates behavioural decisions through the 460 combination of computational modelling of behaviour and identification of neurophysiological 461 signatures of latent variables, in distributed cortical networks. Beta power reflects the temporal and 462 spatial dynamics of the accumulation and transfer of decision-evidence, with a continuous flow of 463 information between regions rather than sequential discrete decisions. During this flow, the gradual 464 transition from the resolution of sensory uncertainty to the resolution of response uncertainty, enables 465 goal-directed actions in the face of uncertainty. 


\section{Participants}

468

469

470

471

472

473

474

475

476

477

478

479

480

481

482

483

484

485

486

487

488

489

490

491

492

493

494

495

496

Twenty healthy volunteers (9 females, 11 males, age range 18-39 years) took part in this study, after providing informed consent. Inclusion criteria included age 18-40 years, right-handed, and screening for neurological or psychiatric illness. Two subjects failed to reach the requisite performance criterion during training and were excluded, leaving 18 subjects in all subsequent behavioral and neural analyses. The chosen sample size was based on previous EEG/MEG studies on perceptual and motor decisionmaking ${ }^{35,66}$. Experimental protocols conformed to the guidelines of the Declarations of Helsinki and were approved by the local research ethics committee.

\section{Task and procedures}

Participants performed a finger-tapping task adapted from previous studies ${ }^{13,14}$. Their goal was to detect the onset of coherent motion and to press the button corresponding to one of the downward moving stimuli (coherent stimuli). The number of coherent stimuli defined two trial types: Low action uncertainty trials, where a single coherent stimulus commanded which button to press; and high action uncertainty trials, where three coherent stimuli required the participants to make a simple choice and press any one of the three corresponding buttons (a "fresh choice, regardless of what you have done in previous trials" ${ }^{\prime 14}$ ). Equal emphasis was placed on the speed and accuracy of the responses. Participants were instructed to fixate on a central red mark throughout the trial. Eye-tracking data collected during the first six scanning sessions confirmed participants were able to successfully perform the task while maintaining fixation (see SI Appendix, Fig. S5). Each trial started with the presentation of the fixation mark and stimuli onset ensued after a variable interval comprised between $0.5 \mathrm{sec}$ and $1 \mathrm{sec}$. The imaging session was preceded by one training psychophysical session and one test session scheduled on separate days; the scanning session was conducted a maximum of four days after the psychophysical training, depending on the availability of the participants. The test session was to ensure that the participants were able to perform well under the individually adjusted motion thresholds. In the test and scan sessions, coherence levels were fixed to the individual thresholds corresponding to high and low levels of perceptual uncertainty, the match-to-sample task was removed, and no feedback was provided except for too late or too long responses. Levels of perceptual and action uncertainty where randomly interspersed across trials. Each session consisted of 10 blocks (total 720 trials per participant) separated by a short rest. Trials on which responses were made before 0.1 -sec or after 2 -sec (on average $1.3 \%$ of total trials) were excluded from subsequent analyses. 
497

498

499

500

501

502

503

504

505

506

507

508

509

510

511

512

513

514

515

516

517

518

519

520

521

522

523

524

525

526

527

\section{Stimuli}

Stimuli were presented using Matlab (Matworks, Natiek, MA, USA) and the Psychotoolbox routines in a sound-proof and dimly lit room. For the psychophysical training stimuli were displayed on a CRT monitor at $60 \mathrm{~cm}$, and for the scan session stimuli were projected on a screen through a projector at $130 \mathrm{~cm}$ (both with a $60 \mathrm{~Hz}$ refresh rate) with equivalent pixel resolution of $0.03^{\circ}$.

Stimuli were four random dot kinematograms displayed within four circular apertures $\left(4^{\circ}\right.$ diameter $)$ positioned along a notional semi-circular arc ( $3.4^{\circ}$ eccentricity) on a black background ( $100 \%$ contrast). 200 dots were displayed during each frame and spatially displaced in the next frame to introduce apparent downward motion ( $6 \% \mathrm{sec}$ velocity). To manipulate motion strength (i.e. motion coherence) between trials, on each frame only a certain proportion of dots moved downward whilst the rest of the dots where randomly reallocated. Motion coherence level was kept constant throughout the trial.

Since abrupt stimulus onset and offset could elicit large sensory-evoked potentials which might mask decision processes, the 1.5 seconds long coherent motion interval was preceded and followed by intervals of zero-coherence levels lasting $1 \mathrm{sec}$ and $0.5 \mathrm{sec}$, respectively.

\section{Psychometric calibration}

Participants were firstly familiarized with the finger-tapping task during a short practice session where $100 \%$ coherent stimuli were adopted. The familiarization phase was completed when participants reached $90 \%$ accuracy across all trial types. In the following psychophysical training, motion coherence was randomly varied between trials to estimate individual motion thresholds. Eight logarithmically spaced motion coherence levels $\left(\begin{array}{llll}0 & 0.5 & 0.10 \ldots . .9\end{array}\right)$ were used (32 trials per level) following extensive piloting to ensure coverage of a wide range of individual motion sensitivity. Each training session comprised 16 blocks of 32 trials. Feedback was provided for correctness of responses as well as for too early or too late responses $(100 \mathrm{~ms}$ and $2.5 \mathrm{~s}$ from motion coherence onset, respectively).

To ensure that participants perceived all the available options (i.e. coherent stimuli) before committing to a decision, occasionally $(\mathrm{p}=0.2)$ after a correct choice they had to perform a secondary match-tosample task: a set of grey discs replaced the stimuli and participants had to report whether their locations matched the location of the previously displayed coherent stimuli. They had to press any button to report a match and withhold any response otherwise. A trial was considered as correct only when both choice and matching were correct. Trials with un-matching responses were discarded and repeated within the session. 
528 To tailor the sensory evidence to the participants' individual motion sensitivity across number of

529 options, the discrimination accuracy of each trial type in each training session was fitted using a 530 maximum likelihood method, with a Log-Quick function defined as

$531 \quad F_{l o g}=1-2^{-10^{\beta(x-\alpha)}}$,

532 where $\alpha$ is the threshold, $\beta$ is the slope and $\mathrm{x}$ is the coherence level. To obtain the proportion correct for 533 each trial type, the Log-Quick function was scaled by,

$534 P=\gamma+(1-\gamma-\lambda) F_{l o g}$,

535 where $\gamma$ is the guess rate and $\lambda$ is the lapse rate controlling the lower and upper asymptote of the 536 psychometric function, respectively.

537 Individual low and high perceptual uncertainty levels for each trial type were estimated as the $75^{\text {th }}$ and $53890^{\text {th }}$ percentile of the psychometric functions from the last session. The reason for adopting these 539 thresholds was twofold: firstly, participants need to perceive all the available options before committing 540 to a decision. Secondly, supra-threshold trials are best suited for investigating neural correlates of 541 evidence accumulation ${ }^{67}$.

\section{MEG and EEG data acquisition and processing}

543 An Elekta Neuromag Vectorview System (Helsinki, Finland) simultaneously acquired magnetic fields from 102 magnetometers and 204 paired planar gradiometers, and electrical potential from 70 EEG electrodes (70 Ag-AgCl scalp electrodes in an Easycap - GmbH, Herrsching, Germany - extended 10$10 \%$ system). Additional electrodes provided a nasal reference, a forehead ground, paired horizontal and vertical electro-oculography, electrocardiography and neck electromyography. All data were recorded and digitized continuously at a sample rate of $1 \mathrm{kHz}$ and high-pass filtered above $0.01 \mathrm{~Hz}$. electrodes location, and the position of three anatomical fiducial points (nasion and left and right preauricular) were recorded using a 3D digitizer (Fastrak Polhemus Inc., Colchester, VA). The initial impedence of all EEG electrodes was optimized to below $10 \mathrm{k} \Omega$, and if this could not be achieved in a 553 particular channel, or if it appeared noisy to visual inspection, it was excluded from further analysis. 554 The 3D position of the head position indicators relative to the MEG sensors was monitored throughout 555 the scan. 
556

557

558

559

560

561

562

563

564

565

566

567

568

569

570

571

572

573

574

575

576

577

578

579

580

581

582

583

584

585

586

587

Environmental noise suppression, motion compensation, and Signal Source Separation for the MEG data was followed by separate independent component analysis for the three sensor types and artifactual components were rejected. For EEG data, components temporally and spatially correlated to eye movements, blinks and cardiac activity were automatically identified with EEGLab's toolbox ADJUST (Swartz Center for Computational Neuroscience, University of California San Diego). For MEG data, components were automatically identified that were both significantly temporally correlated with electrooculography and electrocardiography data, and spatially correlated with separately acquired topographies for ocular and cardiac artifacts using in-house Matlab code.

The continuous artefact-corrected data were low-pass filtered (cut-off $=100 \mathrm{~Hz}$, Butterworth, fourth order), notch filtered between 48 and $52 \mathrm{~Hz}$ to remove main power supply artifacts, down-sampled to $250 \mathrm{~Hz}$, and epoched from -1500 to $2500 \mathrm{~ms}$ relative to motion coherence onset. EEG data were referenced to the average over electrodes.

MEG and EEG data were combined before inversion into source space ${ }^{21}$ using the Miminum Norm algorithm as implemented by SPM12. Notably, combined MEG and EEG allows a better localization of neural sources than each technique on its $\mathrm{own}^{21}$. The forward model was estimated from each participant's anatomical T1-weighted MRI image. All conditions were included in the inversion to ensure an unbiased linear mapping. The source images were spatially smoothed using an $8 \mathrm{~mm}$ FWHM Gaussian kernel.

\section{Accumulator model of perceptual and action decisions}

We adopted formal models of decision-making to decompose the behavioral performance into cognitively relevant latent variables. We fitted accumulation-to-threshold models (Linear Ballistic Accumulator $^{23}$, LBA), to each participant's reaction time and accuracy data. The LBA model of decisions is more tractable than drift-diffusion models for $n$-way decisions while still remaining physiologically informative ${ }^{68}$ and has been previously applied to a finger tapping task to model fMRI evidence accumulation ${ }^{13}$. Instead of adopting a two-stage model, which assumes a discrete serial process between perceptual and action decisions, we opted for a 'unitary' model where both perceptual and action uncertainty concur in determining participant's performance in a given trial. The factorial design of the experiment enabled us to divorce perceptual and action decision processes using connectivity metrics.

According to this class of models, each accumulator linearly integrates the decision-evidence (or the intention) over time in favor of one action, and the decision is made when the accumulated activity reaches threshold (see Fig.3c). In our task possible actions correspond to a button press from one of 
588

589

590

591

592

593

594

595

596

597

598

599

600

601

602

603

604

605

606

607

608

609

610

611

612

613

614

615

616

four fingers, each modeled by independent accumulators $i \in\{1,2,3,4\}$. When three valid actions are available, three accumulators are engaged with activation starting at levels independently drawn from a uniform distribution [0, c0], and increasing linearly over time with an accumulation rate $(v)$ drawn from an independent normal distribution with mean $\mu_{\mathrm{i}}$ and standard deviation $\sigma_{\mathrm{i}}$. A response is triggered once one accumulator wins the 'race' and reaches a decision bound (b). When only one action is available, only the accumulator corresponding to the available action is engaged. Predicted reaction time (RT) is given by the duration of the accumulation process for the winning accumulator, plus a constant non-decision time $t_{0}$ representing the latency associated with stimulus encoding and motor response initiation ${ }^{23}$.

To identify the combinations of free parameters that best accounted for the observed behavioral data we firstly fitted 15 variants (i.e. all possible combinations without repetition) of the LBA. Each variant was characterized by a unique combination of free parameters allowed to vary across trials. The bestfitting parameters for each model variant were used to calculate the Bayesian Information Criterion (BIC), which penalizes extra free parameters in favor of simpler models. BIC values were then used to compare the goodness-of-fit of each variant using random-effects Bayesian model comparison ${ }^{24,25}$. Predictions of decision-related activity were generated from the winning LBA model to locate neural signatures of decisions-evidence accumulation in single-trial analyses of MEEG data.

\section{Estimation of expected neural activity}

We generated predictions of decision-related activity from the LBA model ${ }^{13}$ to locate neural signatures of decisions-evidence accumulation in single-trial analyses of MEEG data. For multiple options, the LBA model assumes multiple active accumulators, one for each finger option. Let $\tilde{\mu}_{W}$ be the accumulation rate of the winning option (i.e. the one reaching response threshold $b$ ), sampled from the normal distribution $N\left(\mu_{W}, \sigma_{W}^{2}\right)$. Let $\tilde{\mu}_{L 1}$ and $\tilde{\mu}_{L 2}$ be the sampled accumulation rates of the alternative options (i.e. the losers), sampled from normal distributions $N\left(\mu_{L 1}, \sigma_{L 1}^{2}\right), N\left(\mu_{L 2}, \sigma_{L 2}^{2}\right)$, respectively. If the reaction time of a given trial is $\mathrm{RT}$, the latency of the accumulation process is $R T-t_{0}$, such that the expected accumulation of the winning option is:

$E\left[\tilde{\mu}_{W}\right]=\frac{b-c_{0} / 2}{R T-t_{0}}$

Since the losing accumulators have not reached the threshold by the time of the response RT, the expected values of $\tilde{\mu}_{L 1}$ and $\tilde{\mu}_{L 2}$ are smaller than $\tilde{\mu}_{W}$. 
617 Therefore, the losing accumulation rates have truncated normal distributions with an upper bound of

$618 \tilde{\mu}_{W}$ and with expected values of:

620

619

$$
\left\{\begin{array}{l}
E\left[\tilde{\mu}_{L 1} \mid \tilde{\mu}_{L 1}<\tilde{\mu}_{W}\right]=\mu_{L 1}-\sigma_{L 1}\left[\frac{\varphi\left(\frac{\widetilde{\mu}_{W}-\mu_{L 1}}{\sigma_{L 1}}\right)}{\Phi\left(\frac{\tilde{u}_{W}-\mu_{L 1}}{\sigma_{L 1}}\right)}\right], \\
E\left[\tilde{\mu}_{L 2} \mid \tilde{\mu}_{L 2}<\tilde{\mu}_{W}\right]=\mu_{L 2}-\sigma_{L 2}\left[\frac{\varphi\left(\frac{\tilde{\mu}_{W}-\mu_{L 2}}{\sigma_{L 2}}\right)}{\Phi\left(\frac{\widetilde{\tilde{W}}_{W}-\mu_{L 2}}{\sigma_{L 2}}\right)}\right]
\end{array}\right.
$$

621 where $\varphi(x)=\frac{1}{\sqrt{2 \pi}} e^{-x^{2} / 2}$ and $\Phi(x)=\frac{1}{\sqrt{2 \pi}} \int_{-\infty}^{x} e^{-x^{2} / 2} d x$

622 The sum of the winning and losing accumulation rates gives an estimation of total accumulation

623 activity for single trials. For trials with only one available option, the accumulation activity is

624 determined by the only active accumulator.

\section{Dimensionality reduction}

626 To improve computational efficiency, reduce multiple comparisons issues while retaining the maximum 627 amount of information ${ }^{69}$, we reduced the dimensionality of the MEEG data by parcellating the cortical 628 surface into a set of 96 regions of interest (ROIs) defined using the Harvard-Oxford cortical atlas (FSL, 629 FMRIB, Oxford) and by representing the dynamic of each ROI with a single time-course, obtained 630 using principal component analysis ${ }^{70}$. The reconstructed sources within each ROI were first bandpass-

631 filtered in either beta $(13-30 \mathrm{~Hz})$ or gamma $(31-90 \mathrm{~Hz})$ frequency bands. The coefficients of the 632 principal component accounting for the majority of the variance of the vertices within each ROI, were 633 then taken as an appropriate representation of source activity for that region. Next, to estimate the power 634 oscillations on a single-trial basis, we extracted frequency-specific signal envelope modulations using 635 a Hilbert transform of the source data from each reconstructed ROI (epochs from 500ms before to $6361500 \mathrm{~ms}$ after coherence onset). The Hilbert's envelope is a convenient measure of how the power of 637 the signal varies over time in the frequency range of interest, and thus particularly suited to capture 638 relatively slow fluctuations associated to the instantaneous accumulation of evidence/intentions. The 639 power estimates of individual participants were down-sampled to $100 \mathrm{~Hz}$ and normalized by their 640 baseline (from $400 \mathrm{~ms}$ to $100 \mathrm{~ms}$ before coherence onset). 


\section{Single-trial analysis}

642 To identify the spatio-temporal profile of decision-related accumulation over the brain we derived

643 model-predicted signals for each trial to compare with neural oscillations in beta and gamma frequency

644 bands. We estimated the maximum lagged absolute Spearman correlation between the model predicted 645 activity and the signal envelope in a trial-by-trial fashion. The lagged correlation was used to optimally

646 split the non-decision time before and after the accumulation period to determine the time delay between

647 the neural signal and the model predictions. The time before accumulation provides a measure of the

648 temporal separation between coherence onset and accumulation onset.

649 If the model prediction $x$ is a lagged version of the neural signal $y$ so that

$650 y(t)=x\left(t+\tau_{0}\right)$

651 Where $\tau_{0}$ is a time delay that can vary from $0 \mathrm{~ms}$ to the individual non-decision time $\left(t_{0}\right)$ with steps of

$65210 \mathrm{~ms}$, then the maximum absolute lagged correlation between $x$ and $y$ is defined as

$653 \rho_{x y}(t)=\max \left|\operatorname{corr}\left(y(t), x\left(t-\tau_{i}\right)\right)\right|$,

654 where $i=\left[0,10,20 \ldots t_{0}\right]$.

655 With the peak value of $\rho_{x y}(t)$ occurring when $\tau_{i}=\tau_{0}$ which allows us to determine the time delay.

656 We estimated the largest absolute lagged correlation value for each ROI and individuals by comparing 657 concatenated epochs and model predictions. This choice permits to measure accumulation lags specific 658 to each ROI, under the assumption that they differ across brain regions for each participant. The 659 strength of the Fisher-transformed maximum lagged correlations for each ROI was then quantified (zscore) using a one-sample sign-test. To provide a conservative estimate of significant correlations between model prediction and neural activity, we repeated the above procedure 10.000 times, each iteration using a different phase-randomized version of the original MEEG signal, to obtain a distribution of correlations under chance. Two-tailed statistical significance was assessed by computing the proportion of absolute values of the distribution of correlations generated by chance exceeding the correlation between model predictions and the original MEEG signal. The resulting p-values were corrected for multiple comparisons (False Discovery Rate) across ROIs and frequency bands.

\section{Connectivity analyses}

To explore the direction of the information flow we employed phase-transfer entropy, a data-driven 
670

671

672

673

674

675

676

677

678

679

680

681

682

683

684

685

686

687

688

689

690

691

\section{2}

693

694

695

696

697

698

between ROIs whose activity best matched with model's predicted activity was estimated using the directed phase-transfer entropy. The analyses focused on regions whose activity significantly fitted the LBA model's prediction.

To identify the ROIs that preferentially accumulated evidence for perception or action decisions, the average information flow (quantified by phase transfer entropy) sent by each ROI was calculated for each subject and condition. The difference of information flow between uncertainty levels for perception and action is compared at the ROI level with a surrogate distribution generated by flipping the condition labels for a random number of participants (10.000 iterations). Since significance was estimated separately for perception and action, the critical value for the FDR correction was halved to $\alpha=0.025$.

To quantify the direction of information flow, we calculated a posterior to anterior index (PAx) as implemented by Hillebrand et al, 2016. A positive PAx indicates preferential flow from posterior regions toward anterior regions. ROIs were split into anterior and posterior region with respect to the central sulcus (see SI Appendix, Table S2). Significance was assessed with permutation testing where the average directional phase-transfer entropies were shuffled across ROIs and PAx was estimated. This procedure was repeated 10.000 times to generate a surrogate distribution of PAx values against which the observed PAx values were tested ( $\mathrm{p}<0.025$ to account for multiple comparisons).

For the correlations in Fig. 7a, we first confirmed homoscedasticity of our data and then calculated bootstrapped Pearson's correlations. For the correlations in Fig. 7b we used repeated-measures correlation (as implemented in the rmcorr package in R) which accounts for non-independence among observations due to multiple measurements per participant. The resulting p-values were corrected for multiple comparisons by applying Holm-Bonferroni correction.

\section{Hypothesis testing}

Differences in reaction times were tested with a 2-way repeated measures ANOVA (Low/High Uncertainty x Action/Perception). All other hypothesis tests used non-parametric tests or random permutation methods that do not rely on specific assumptions about the distributions of data values. All tests were evaluated at the $\mathrm{p}<0.05$ level (two-tailed), correcting for multiple comparisons where appropriate. 
700

701

702

703

704

705

706

707

708

709

710

711

712

713

714

715

716

717

718

719

720

721

722

723

724

725

726

727

728

729

730

731

732

\section{Data and code availability}

Additional data related to this study are available from the corresponding author upon request. The code and data to generate the results and the figures of this study are available at https://github.com/ale-tom/MEEG Uncertainty.

\section{References}

1. Humphreys, G. W. et al. Attending to waht you are doing: Neuropsychological and experimental evidence for interactions between perception and action. in Attention in action 3 25 (2004).

2. Milner, A. D. et al. Perception and action in 'visual form agnosia'. Brain 114 ( Pt 1, 405-28 (1991).

3. Crutch, S. J., Yong, K. X. X. \& Shakespeare, T. J. Looking but Not Seeing: Recent

4. Philiastides, M. G. \& Sajda, P. EEG-Informed fMRI Reveals Spatiotemporal Characteristics of Perspectives on Posterior Cortical Atrophy. Curr. Dir. Psychol. Sci. 25, 251-260 (2016).

5. Rae, C. L., Hughes, L. E., Weaver, C., Anderson, M. C. \& Rowe, J. B. Selection and stopping in voluntary action: a meta-analysis and combined fMRI study. Neuroimage 86, 381-91 (2014).

6. Rushworth, M. F. S., Noonan, M. P., Boorman, E. D., Walton, M. E. \& Behrens, T. E. Frontal Cortex and Reward-Guided Learning and Decision-Making. Neuron 70, 1054-1069 (2011).

7. Wang, X. J. Probabilistic decision making by slow reverberation in cortical circuits. Neuron 36, 955-968 (2002).

8. Zhang, J. The effects of evidence bounds on decision-making: Theoretical and empirical developments. Front. Psychol. 3, 1-19 (2012).

9. Wald, A. \& Wolfowitz, J. Bayes Solutions of Sequential Decision Problems. Ann. Math. Stat. 21, 82-99 (1950).

10. Gold, J. I. \& Shadlen, M. N. The neural basis of decision making. Annu. Rev. Neurosci. 30, 535-74 (2007).

11. Roitman, J. D. \& Shadlen, M. N. Response of neurons in the lateral intraparietal area during a combined visual discrimination reaction time task. J. Neurosci. 22, 9475-9489 (2002).

12. Pesaran, B., Nelson, M. J. \& Andersen, R. A. Free choice activates a decision circuit between frontal and parietal cortex. Nature 453, 406-409 (2008). 
733

734

735

736

737

738

739

740

741

742

743

744

745

746

747

748

749

750

751

752

753

754

755

756

757

758

759

760

761

762

763

764

765

766

767

768

769

13. Rowe, J. B., Hughes, L. \& Nimmo-Smith, I. Action selection: a race model for selected and non-selected actions distinguishes the contribution of premotor and prefrontal areas.

Neuroimage 51, 888-96 (2010).

14. Zhang, J., Hughes, L. E. \& Rowe, J. B. Selection and inhibition mechanisms for human voluntary action decisions. Neuroimage 63, 392-402 (2012).

15. Sternberg, S. High-Speed Scanning in Human Memory. Science (80-. ). 153, 652-654 (1966).

16. McClelland, J. L. On the time relations of mental processes: An examination of systems of processes in cascade. Psychol. Rev. 86, 287-330 (1979).

17. Polanía, R., Krajbich, I., Grueschow, M. \& Ruff, C. C. Neural Oscillations and Synchronization Differentially Support Evidence Accumulation in Perceptual and ValueBased Decision Making. Neuron 82, 709-720 (2014).

18. Bonaiuto, J. J. et al. Lamina-specific cortical dynamics in human visual and sensorimotor cortices. Elife 1-32 (2018). doi:10.7554/eLife.33977

19. Hughes, L. E., Rittman, T., Robbins, T. W. \& Rowe, J. B. Reorganization of cortical oscillatory dynamics underlying disinhibition in frontotemporal dementia. Brain 141, 24862499 (2018).

20. Haegens, S., Vergara, J., Rossi-Pool, R., Lemus, L. \& Romo, R. Beta oscillations reflect supramodal information during perceptual judgment. Proc. Natl. Acad. Sci. 114, 13810-13815 (2017).

21. Henson, R. N., Mouchlianitis, E. \& Friston, K. J. MEG and EEG data fusion : Simultaneous localisation of face- evoked responses. Neuroimage 47, 581-589 (2010).

22. Hillebrand, A. et al. Direction of information flow in large-scale resting-state networks is frequency-dependent. Proc. Natl. Acad. Sci. 113, 3867-3872 (2016).

23. Brown, S. D. \& Heathcote, A. The simplest complete model of choice response time: Linear ballistic accumulation. Cogn. Psychol. 57, 153-178 (2008).

24. Stephan, K. E., Penny, W. D., Daunizeau, J., Moran, R. J. \& Friston, K. J. Bayesian model selection for group studies. Neuroimage 46, 1004-17 (2009).

25. Tomassini, A., Ruge, D., Galea, J. M., Penny, W. \& Bestmann, S. The role of dopamine in temporal uncertainty. J. Cogn. Neurosci. 28, (2016).

26. Resulaj, A., Kiani, R., Wolpert, D. M. \& Shadlen, M. N. Changes of mind in decision-making. Nature 461, 263-266 (2009).

27. Caballero, J. A., Humphries, M. D. \& Gurney, K. N. A probabilistic, distributed, recursive mechanism for decision-making in the brain. PLoS Computational Biology 14, (2018).

28. Park, I. M., Meister, M. L. R., Huk, A. C. \& Pillow, J. W. Encoding and decoding in parietal cortex during sensorimotor decision-making. Nat. Neurosci. 17, 1395-1403 (2014).

29. Donner, T. H., Siegel, M., Fries, P. \& Engel, A. K. Buildup of Choice-Predictive Activity in Human Motor Cortex during Perceptual Decision Making. Curr. Biol. 19, 1581-1585 (2009). 
30. Kubanek, J., Snyder, L. H., Brunton, B. W., Brody, C. D. \& Schalk, G. A low-frequency oscillatory neural signal in humans encodes a developing decision variable. Neuroimage $\mathbf{8 3}$, 795-808 (2013).

31. Gould, I. C., Nobre, a. C., Wyart, V. \& Rushworth, M. F. S. Effects of Decision Variables and Intraparietal Stimulation on Sensorimotor Oscillatory Activity in the Human Brain. $J$. Neurosci. 32, 13805-13818 (2012).

32. Donner, T. H. et al. Population Activity in the Human Dorsal Pathway Predicts the Accuracy of Visual Motion Detection. J.Neurosci. 98, 345-359 (2007).

33. Kopell, N., Ermentrout, G. B., Whittington, M. A. \& Traub, R. D. Gamma rhythms and beta rhythms have different synchronization properties. Proc. Natl. Acad. Sci. 97, 1867-1872 (2000).

34. Philiastides, M. G., Ratcliff, R. \& Sajda, P. Neural representation of task difficulty and decision making during perceptual categorization: a timing diagram. J. Neurosci. 26, 89658975 (2006).

35. Wyart, V., de Gardelle, V., Scholl, J. \& Summerfield, C. Rhythmic Fluctuations in Evidence Accumulation during Decision Making in the Human Brain. Neuron 76, 847-858 (2012).

36. Kiani, R., Hanks, T. D. \& Shadlen, M. N. Bounded integration in parietal cortex underlies decisions even when viewing duration is dictated by the environment. $J$ Neurosci $\mathbf{2 8}, 3017-$ 3029 (2008).

37. Kerns, J. G. et al. Anterior cingulate conflict monitoring and adjustments in control. Science 303, 1023-6 (2004).

38. Mueller, V. A., Brass, M., Waszak, F. \& Prinz, W. The role of the preSMA and the rostral cingulate zone in internally selected actions. Neuroimage 37, 1354-1361 (2007).

39. Pesaran, B. \& Freedman, D. J. Where Are Perceptual Decisions Made in the Brain? Trends Neurosci. 39, 642-644 (2016).

40. Grinband, J., Hirsch, J. \& Ferrera, V. P. A neural representation of categorization uncertainty in the human brain. Neuron 49, 757-763 (2006).

41. Klaes, C., Westendorff, S., Chakrabarti, S. \& Gail, A. Choosing Goals, Not Rules: Deciding among Rule-Based Action Plans. Neuron 70, 536-548 (2011).

42. Gherman, S. \& Philiastides, M. G. Human VMPFC encodes early signatures of confidence in perceptual decisions. Elife 7, 1-28 (2018).

43. Mendoza-Halliday, D., Torres, S. \& Martinez-Trujillo, J. C. Sharp emergence of featureselective sustained activity along the dorsal visual pathway. Nat. Neurosci. 17, 1255-1262 (2014).

44. Fries, P. Rhythms for Cognition: Communication through Coherence. Neuron 88, 220-235 (2015).

45. Selen, L. P. J., Shadlen, M. N. \& Wolpert, D. M. Deliberation in the Motor System: Reflex Gains Track Evolving Evidence Leading to a Decision. J. Neurosci. 32, 2276-2286 (2012). 
46. Shipp, S. Neural elements for predictive coding. Front. Psychol. 7, 1-21 (2016).

47. Bastos, A. M. et al. Canonical Microcircuits for Predictive Coding. Neuron 76, 695-711 (2012).

48. Friston, K. et al. The anatomy of choice: active inference and agency. Front. Hum. Neurosci. 7, 1-18 (2013).

49. Holmes, C. D., Papadimitriou, C. \& Snyder, L. H. Dissociation of LFP power and tuning in the frontal cortex during memory. J. Neurosci. 38, 3629-17 (2018).

50. Haegens, S. et al. Beta oscillations in the monkey sensorimotor network reflect somatosensory decision making. Proc. Natl. Acad. Sci. 108, 10708-10713 (2011).

51. Haenschel, C., Baldeweg, T., Croft, R. J., Whittington, M. \& Gruzelier, J. Gamma and beta frequency oscillations in response to novel auditory stimuli: A comparison of human electroencephalogram (EEG) data with in vitro models. Proc. Natl. Acad. Sci. 97, 7645-7650 (2000).

52. Traub, R. D., Whittington, M. A., Buhl, E. H., Jefferys, J. G. \& Faulkner, H. J. On the mechanism of the gamma --> beta frequency shift in neuronal oscillations induced in rat hippocampal slices by tetanic stimulation. J. Neurosci. 19, 1088-1105 (1999).

53. Buffalo, E. A., Fries, P., Landman, R., Buschman, T. J. \& Desimone, R. Laminar differences in gamma and alpha coherence in the ventral stream. Proc. Natl. Acad. Sci. 108, 11262-11267 (2011).

54. Bastos, A. M., Loonis, R., Kornblith, S., Lundqvist, M. \& Miller, E. K. Laminar recordings in frontal cortex suggest distinct layers for maintenance and control of working memory. Proc. Natl. Acad. Sci. 115, 1117-1122 (2018).

55. Auksztulewicz, R. et al. Not All Predictions Are Equal: "What" and "When" Predictions Modulate Activity in Auditory Cortex through Different Mechanisms. J. Neurosci. 38, 86808693 (2018).

56. Olufsen, M. S., Whittington, M. A., Camperi, M. \& Kopell, N. New roles for the gamma rhythm: Population tuning and preprocessing for the beta rhythm. J. Comput. Neurosci. 14, 33-54 (2003).

57. Spitzer, B. \& Haegens, S. Beyond the Status Quo: A Role for Beta Oscillations in Endogenous Content (Re-) Activation. Eneuro 4, ENEURO.0170-17.2017 (2017).

58. Allen, W. E. et al. Global Representations of Goal-Directed Behavior in Distinct Cell Types of Mouse Neocortex. Neuron 94, 891-907.e6 (2017).

59. Katz, L., Yates, J., Pillow, J. W. \& Huk, A. Dissociated functional significance of choicerelated activity across the primate dorsal stream. Nature 535, Salt Lake City USA (2016).

60. Siegel, M., Engel, A. K. \& Donner, T. H. Cortical network dynamics of perceptual decisionmaking in the human brain. Front. Hum. Neurosci. 5, 21 (2011).

61. Cisek, P. \& Kalaska, J. F. Neural Mechanisms for Interacting with a World Full of Action Choices. Annu. Rev. Neurosci. 33, 269-298 (2010). 
62. Zhang, J. et al. Different decision deficits impair response inhibition in progressive supranuclear palsy and Parkinson's disease. Brain 139, 161-173 (2016).

63. Sadnicka, A., Daum, C., Meppelink, A. M., Manohar, S. \& Edwards, M. Reduced drift rate: a biomarker of impaired information processing in functional movement disorders. Brain 143, 674-683 (2020).

64. Schnitzler, A. \& Gross, J. Normal and pathological oscillatory communication in the brain. Nat. Rev. Neurosci. 6, 285-296 (2005).

65. Uhlhaas, P. J. \& Singer, W. Neural Synchrony in Brain Disorders: Relevance for Cognitive Dysfunctions and Pathophysiology. Neuron 52, 155-168 (2006).

66. Pape, A. A. \& Siegel, M. Motor cortex activity predicts response alternation during sensorimotor decisions. Nat. Commun. 7, 1-10 (2016).

67. Forstmann, B. U., Ratcliff, R. \& Wagenmakers, E.-J. Sequential Sampling Models in Cognitive Neuroscience: Advantages, Applications, and Extensions. Annu. Rev. Psychol. 67, 641-66 (2016).

68. Cassey, P., Heathcote, A. \& Brown, S. D. Brain and Behavior in Decision-Making. PLoS Comput. Biol. 10, (2014).

69. Kiebel, S. J. \& Friston, K. J. Statistical parametric mapping for event-related potentials: I. Generic considerations. Neuroimage 22, 492-502 (2004).

70. Colclough, G. L., Brookes, M. J., Smith, S. M. \& Woolrich, M. W. A symmetric multivariate leakage correction for MEG connectomes. Neuroimage 117, 439-448 (2015). 